\title{
A. Akhaddar (ed): Atlas of Infections in Neurosurgery and Spinal Surgery
}

\author{
Springer International Publishing Switzerland 2017, 343 pp., 432 figs. of which 315 are in \\ color, hardcover (in English) (ISBN 978-3-319-60085-7, eBook ISBN 978-3-319-60086-4)
}

Mehmet Turgut ${ }^{1}$

Received: 18 September 2017 / Accepted: 9 October 2017 /Published online: 13 October 2017

(C) Springer-Verlag GmbH Germany 2017

The book Atlas of Infections in Neurosurgery and Spinal Surgery covers most common, but still controversial owing to the limited individual experience, infectious conditions seen in neurosurgical and spinal practices and requiring surgical interventions. It is an excellent issue divided in five sections containing a total of 30 chapters: a general introduction, craniocerebral infections, vertebro medullary infections, infections following cranial and spinal surgery, and a section describing the most important specific pathogens and other particular conditions. Each section allows an easy and enjoyable reading and provides a complete reference list useful for readers desiring a deeper insight into a specific subject.

The first two chapters devoted to basic information (classification, sources of infections, laboratory collections, and sample processing), thirteen about infections of the brain and its coverings, five concerning infections of the spine and its coverings, two comprising surgical site infections in cranial and spinal surgeries, and the remainder eight chapters related to specific pathogens and other particular conditions, even though there is some overlapping between various chapters. Each chapter within the book has an epidemiology and etiology, clinical presentation, imaging features, laboratory findings, treatment options, and outcome subsections. In each of them, the involvement of each lesion and area is dealt with in a brief and easy-to-comprehend manner. In particular, each of neuroimaging and laboratory abnormalities is then linked to their clinical features, treatment procedures, and surgical views to encourage a smooth and easy practical integration. At the end of the book, a subject index with appropriate key words facilitates the search of information.

In summary, the book with its 343 pages is easy to hand and it is well documented as a reliable and accurate educational tool, easy to consult and enjoyable to read.

Especially intended for young neurosurgeons, this comprehensive visual encyclopedia can become an excellent reference book also for experienced neurosurgeons as well as neurologists, rheumatologists, radiologists, ENT surgeons, microbiologists, pathologists, and infectious disease specialists.

Compliance with ethical standards

Conflict of interest None.

Ethical statements This review required no ethical approval.

Mehmet Turgut

drmturgut@yahoo.com

1 Department of Neurosurgery, Adnan Menderes University School of Medicine, Aydın, Turkey 